\title{
Comparative genomics and functional roles of the ATP-dependent proteases Lon and Clp during cytosolic protein degradation
}

\author{
Dilip Chandu, Dipankar Nandi *
}

\begin{abstract}
The general pathway involving adenosine triphosphate (ATP)-dependent proteases and ATP-independent peptidases during cytosolic protein degradation is conserved, with differences in the enzymes utilized, in organisms from different kingdoms. Lon and caseinolytic protease (Clp) are key enzymes responsible for the ATP-dependent degradation of cytosolic proteins in Escherichia coli. Orthologs of E. coli Lon and Clp were searched for, followed by multiple sequence alignment of active site residues, in genomes from seventeen organisms, including representatives from eubacteria, archaea, and eukaryotes. Lon orthologs, unlike ClpP and ClpQ, are present in most organisms studied. The roles of these proteases as essential enzymes and in the virulence of some organisms are discussed.
\end{abstract}

Keywords: ATP-dependent proteases; Clp; Comparative and functional genomics; Cytosolic protein degradation; Lon

\section{Introduction}

Cytosolic protein degradation is an active area of investigation and its importance in homeostasis and regulation of important cellular pathways is increasingly appreciated. In general, proteins with well folded structures are stable. Under certain conditions, e.g., unstable proteins, or as proteins age or become damaged, hydrophobic residues that are normally buried are exposed on the surface. Chaperones bind to these hydrophobic residues and attempt to refold these proteins. If refolding is not possible, these misfolded proteins may bind to regulatory components of proteases and undergo degradation. Consequently, cells lacking chaperones or proteases accumulate large amounts of aggregated proteins, demonstrating a close relationship between protein folding and degradation (reviewed in [7,17]).

The cytosolic pathway leading to release of amino acids from an intact protein can be divided into two parts based on the utilization of metabolic energy. The proximal ATPdependent steps are followed by ATP-independent events (Fig. 1a). This basic model of protein unfolding followed by degradation is conserved in eubacteria, archaea and eu- karyotes and involves several steps. First, ATP-dependent enzymes are responsible for the recognition, unfolding and cleavage of substrate proteins into large peptides (Fig. 1a). These enzymes harbor a domain conferring ATPase activity, belonging to the ATPase family associated with various cellular activities (AAA) in the same polypeptide (e.g., Lon) or associated with a different polypeptide-containing ATPase activity (e.g., ClpA, ClpX, ClpY, 19S regulator) (reviewed in [7,17]). Interestingly, the unavailability of tRNAs during polypeptide biosynthesis results in the addition of an eleven-amino-acid peptide tag (AANDENYALAA) encoded by transfer and messenger RNA (tmRNA) to the C-terminus of the truncated polypeptide, and proteins containing tmRNA encoded peptide tags are degraded rapidly (reviewed in [12]). Substrate proteins are unfolded by regulators via conformational changes driven by ATP hydrolysis. Next, unfolded substrate proteins are actively translocated into the proteolytic chambers present in the proteases, which make the initial cuts to generate large peptides. These are cleaved into smaller peptides and, finally, into amino acids, which are recycled into the cellular pool. The ATP-independent steps of the cytosolic protein degradation pathway are often referred to as "downstream processing of peptides" [5].

$26 \mathrm{~S}$ proteasomes (19S regulator $+20 \mathrm{~S}$ proteasomes) are responsible for most cytosolic protein degradation in eukary- 


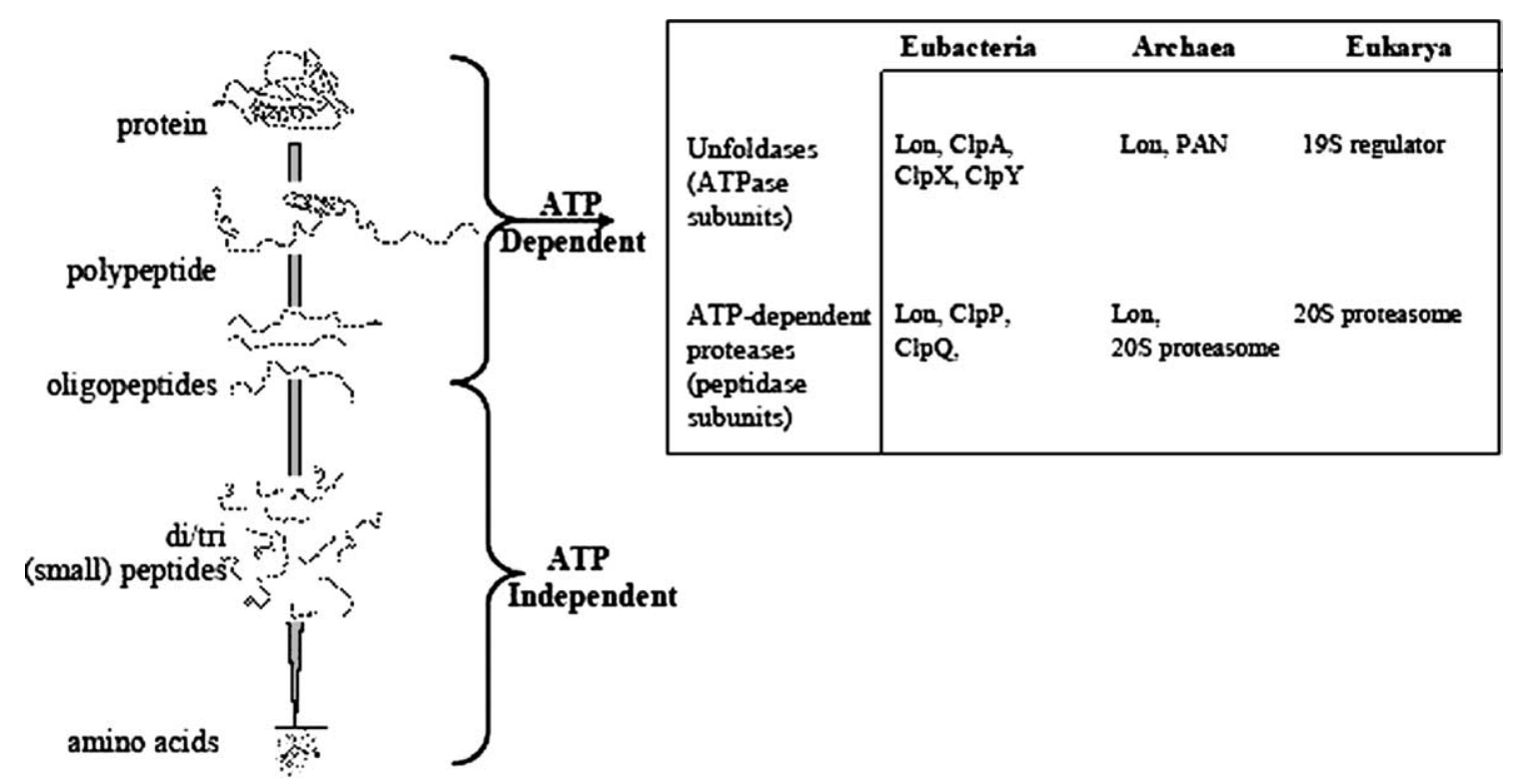

(a)

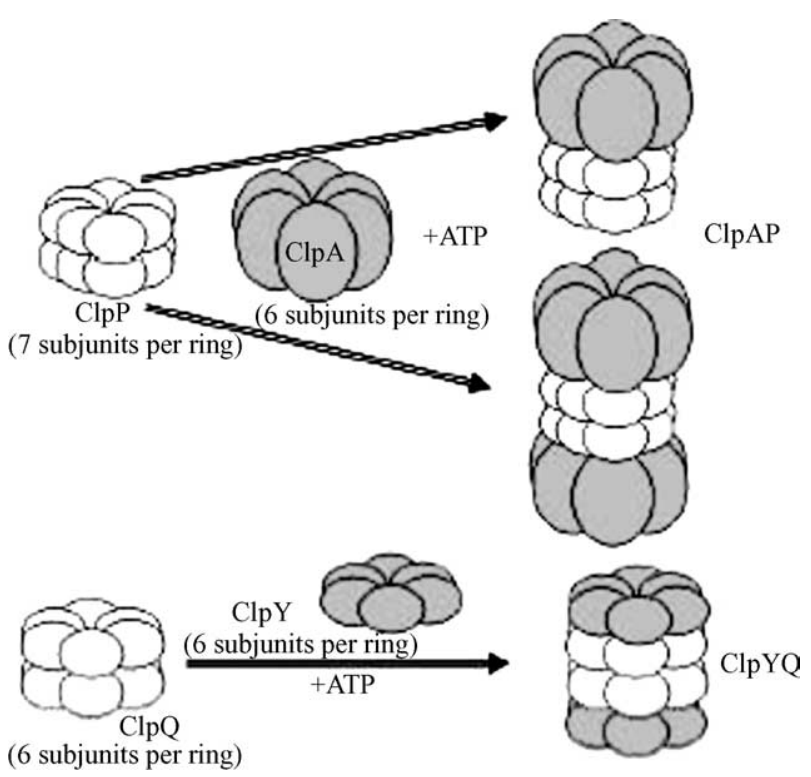

(b)

Fig. 1. General scheme and enzymes involved in cytosolic protein degradation. (a) The intracellular protein degradation pathway leading to the release of free amino acids from intact proteins is represented in this schematic diagram. The initial steps are dependent on ATP-dependent proteases whereas the latter steps are performed by ATP-independent peptidases. The regulatory and protease subunits of ATP-dependent enzymes from different kingdoms are tabulated in the inset. (b) The protease and regulatory components of ClpAP (top) and ClpYQ (bottom) associate and are stable in the presence of ATP to form active complexes that unfold and degrade proteins. Lon harbors both the peptidase and regulatory domains in the same polypeptide and is not shown.

otes. However, Lon and ClpP are the key enzymes responsible for degradation of cytosolic proteins in Escherichia coli. To detect orthologs of these proteases, genome analysis of completed sequences of representative organisms (TBLASTN searches) using full length $E$. coli protease sequences was performed (Table 1). The results of this analysis are represented in Table 1. Furthermore, amino acid sequences important for catalysis in Lon and Clp proteases are discussed. We utilized two criteria to select a particular gene as an ortholog: (i) the E-value depicting the chance factor to be as low as possible (Table 1, parentheses); and (ii) selected orthologs possessing conserved active site residues. Orthologs with a significantly low E-value but lacking active site residues were observed for several enzymes but were not considered for multiple sequence alignment performed using ClustalW. It is possible that some proteins with a high E-value may be important; however, this would require detailed modeling and biochemical studies which do not fall into the scope of this study. In addition, we have reviewed the literature on Lon, Clp proteases and their orthologs with 
Table 1

List of E. coli peptidases selected for whole genome analysis of representative organisms from various branches of the evolutionary tree

\begin{tabular}{|c|c|c|c|c|c|c|c|c|c|c|c|c|c|c|c|c|c|}
\hline & $\mathrm{Ec}$ & Hs & $\mathrm{Mm}$ & At & $\mathrm{Ce}$ & $\mathrm{Dm}$ & $\mathrm{Sc}$ & $\mathrm{Ta}$ & Ss & Bs & $\mathrm{Ll}$ & Mt & Ml & Mge & Mpe & Mpn & $\mathrm{Mpu}$ \\
\hline Lon & $\begin{array}{l}\text { sms } \\
(8 \mathrm{e}-04) \\
\text { b0955 } \\
(0.005)\end{array}$ & $\begin{array}{l}\text { pLon } \\
(\mathrm{e}-139) \\
\text { Lonlike } \\
(\mathrm{e}-138) \\
\text { mLon } \\
(\mathrm{e}-138) \\
\text { mLon1 } \\
\text { (e-136) } \\
\text { Lon- } \\
\text { plantlike } \\
\text { (e-134) }\end{array}$ & $\begin{array}{l}\text { Lon } \\
(\mathrm{e}-140) \\
\text { mLon } \\
(\mathrm{e}-137) \\
\text { mLon } 1 \\
(2 \mathrm{e}-95)\end{array}$ & $\begin{array}{l}\text { nLonm } \\
\text { (e-144) } \\
\text { Lon1 } \\
\text { (e-137) } \\
\text { mLon } \\
\text { (e-136) } \\
\text { Lon } \\
\text { (e-120) } \\
\text { Lonlike } \\
\text { (6e-89) }\end{array}$ & $\begin{array}{l}\text { mLon } \\
(\mathrm{e}-136) \\
\text { Lon } \\
(\mathrm{e}-115)\end{array}$ & $\begin{array}{l}\text { LD30525 } \\
\text { (e-148) } \\
\text { CD8798 } \\
(\mathrm{e}-127)\end{array}$ & $\begin{array}{l}\text { PIM1 } \\
\text { (e-133) }\end{array}$ & $\begin{array}{l}\text { Lon } \\
(6 \mathrm{e}-20)\end{array}$ & & $\begin{array}{l}\text { LonA } \\
(0.0)\end{array}$ & & & & $\begin{array}{l}\text { Lon } \\
\text { (e-157) }\end{array}$ & $\begin{array}{l}\text { Lon } \\
(\mathrm{e}-169)\end{array}$ & $\begin{array}{l}\text { Lon } \\
\text { (e-161) }\end{array}$ & $\begin{array}{l}\text { Lon } \\
\text { (e-147) }\end{array}$ \\
\hline ClpP & & $\begin{array}{l}\text { ClpP } \\
(1 \mathrm{e}-49)\end{array}$ & $\begin{array}{l}\text { ClpP } \\
(4 \mathrm{e}-66)\end{array}$ & $\begin{array}{l}\text { ClpP1 } \\
(9 \mathrm{e}-61) \\
\text { nClpP1 } \\
(4 \mathrm{e}-58) \\
\text { nClpP3 } \\
(4 \mathrm{e}-50) \\
\text { nClpP4 } \\
(3 \mathrm{c}-58)\end{array}$ & $\begin{array}{l}\text { ClpP } \\
(3 e-56)\end{array}$ & $\begin{array}{l}\text { CG5045 } \\
(4 \mathrm{e}-61)\end{array}$ & & & & $\begin{array}{l}\text { ClpP } \\
(5 \mathrm{e}-74)\end{array}$ & $\begin{array}{l}\text { ClpP } \\
(2 \mathrm{e}-60)\end{array}$ & $\begin{array}{l}\text { ClpP } \\
(1 \mathrm{e}-49) \\
\text { ClpP2 } \\
(3 \mathrm{e}-49)\end{array}$ & $\begin{array}{l}\text { ClpP } \\
(3 e-49) \\
\text { ClpP2 } \\
(4 e-49)\end{array}$ & & & & \\
\hline ClpQ & & & & & & & & & & $\begin{array}{l}\text { HslV } \\
(1 \mathrm{e}-40)\end{array}$ & & & & & & & \\
\hline
\end{tabular}

Numbers in parentheses represent the E-value, reflecting the significance of the similarity between the homologue and the respective E. coli peptidase.

The abbreviations used are as follows: Ec, Escherichia coli; Hs, Homo sapiens; Mm, Mus musculus; At, Arabidopsis thaliana; Ce, Caenorahditis elegans; Dm, Drosophila melanogaster; Sc, Saccharomyces cerevisiae; Ta, Thermoplasma acidophilum; Ss, Sulfolobus solfataricus; Bs, Bacillus subtilis; L1, Lactococcus lactis; Mt, Mycobacterium tuberculosis; Ml, Mycobacterium leprae; Mge, Mycoplasma genetalium; Mpe, Mycoplasma penetrans; Mpn, Mycoplasma pneumoniae; Mpu, Mycoplasma pulmonis.

respect to their structures and roles of these proteases in growth and virulence. Proteases involved in cytosolic protein degradation in bacteria recognize different substrates with overlapping specificities [48]. In fact, E. coli lacking three proteases, lon, $\operatorname{clp} P$ and $\operatorname{clp} Q$, but not a single protease, is required to prevent the degradation of homoserine trans-succinylase, the first enzyme involved in biosynthesis of methionine [1]. A catalogue of some key in vitro and in vivo substrates hydrolyzed by Lon, ClpP and ClpQ is presented in Table 2.

\section{Lon}

Lon protease is a multimer with each subunit $(87 \mathrm{kDa})$ having three distinct domains: an N-terminal domain with an unassigned function, a central domain with ATPase activity and the C-terminal proteolytic domain harboring the active site serine (Ser-679). In eukaryotes, Lon paralogs are present in the mitochondrial matrix, peroxisomes or plastids. Cryo-electron microscopy has demonstrated that yeast mitochondrial Lon exists as a ring-shaped heptamer [38]. Lon and its orthologs display a conserved ATP binding motif "GPPGVGKT" in the central domain and ATP binding presumably causes a conformational change. In fact, in the absence of ATP, yeast mitochondrial Lon consists of asymmetric rings whereas ATP binding results in formation of symmetric rings [38].
Comparison of Lon amino acid sequences from different sources revealed the presence of a Ser-Lys catalytic dyad. Furthermore, site-specific mutagenesis of the Ser-Lys catalytic dyad residues demonstrated that these were essential for catalysis in VP4, a Lon ortholog encoded by an infectious bursal disease virus strain P2 [2]. Recently, the crystal structure of the proteolytic domain of E. coli Lon revealed the presence of a unique fold and modeling of the active site demonstrated the close association of Lys-722 with Ser-679 [4]. Interestingly, the Lon Ser-679 mutant, but not an ATPase domain mutant, is able to complement UV sensitivity and capsule overproduction in a strain lacking Lon activity, probably due to substrate sequestration [44]. This apparent complementation suggests that substrate binding followed by unfolding and proteolysis are coupled events.

Lon orthologs are widely conserved in different kingdoms (Table 1). Residues corresponding to the catalytic dyad, Ser-679 and Lys-722, in E. coli Lon, are present in most orthologs (Fig. 2a). Archaeabacterial representatives showed mixed distribution. Sulfolobus, a crenarchaeote, does not harbor a significant Lon ortholog, unlike the euryarchaeote member Thermoplasma (Table 1, Fig. 2). As shown in Fig. 2b, the T. acidophilum Lon is not closely related to E. coli Lon and further biochemical analysis is required. Lon orthologs are present in Methanococcus jannaschii, Pyrococcus furiosus, Methanosarcina mazei and Halobacterium sp. NRC-1 (data not shown), suggesting that Lon is fairly well distributed in archaea. In fact, Lon from the 
E.coli_Lon

B. subtilis LonA.

M.pneumoniāe_Lon

M.genitalium_Lon

M. penetrans_Lon

M.pulmonis Lon

M.musculus Lon

M.musculus_mLon1

H. sapiens pLon

H. sapiens Lon-plant I

H.sapiens_mLon1

A.thalianā_Lon1

H. sapiens Lonlike

H. sapiens mLon

M.musculus mLon

D.mel anog aster_LD30525

D.mel anog aster CD8798

C.elegans mLon

A.thalianānnLonm

A. thaliana-mLon

A.thaliana Lon

A. thaliana LonLike

S.cerevisiāe_PIM1

C. elegans Lon

B. subtilis LonB

T. acidophi $\bar{l}$ um_Lon

E.coli sms

E.coli b0955
PDFYEKRDIHVHVPEGATPKDGPSAG IAMCTALVSCLTGNPVRADVAMTGEITLRGQVLPIGGLKEKLL-------AAHRGGI 731 PDFHEKYDIHIHVPEGAVPKDGPSAG ITMATALVSALTGRAVSREVGMTGEITLRGRVLPIGGLKEKAL-------GAHRAGL 729 PNLF KKVDINIHVPGGG IPKDG PSAGAALVTAIISSLTGKKVDPKIAMTGE ITLRGKVMTIGGVKEKTI-------SAYRGGV 754 PSLEKKIDINIHVPGGG IPKDG PSAGAALVTAIISSLTGKKVDPTVAMTGE ITLRGKVLVIGGVKEKTI-------SAYRGGV 754 PKIFGE IDLHVHVPSGG IPKDG PSAG IALTTAILSALKNVKI PSNVAMTGE ITLRGRVLIIGGVKEKTI-------SAYRGGA 740 DFDWENNSIHIHVPDGATPKDGPSAGVTETTAIISALTKKKVSPLVGMTGE ITLRGKVLPIGGLKEKSI-------AANKFGI 799 FDLLDNTDIHLHF PAGAVTKDG PSAGVT IVTCLASLF SGRLVRSDVAMTGE ITLRGLVLPVGGIKDKVL-------AAHRAGL 795 FDLLDNTDIHLHF PAGAVTKDG PSAGVT IVTCLASLF SGRLVRSDVAMTGE ITLRGLVLPVGGIKDKVL-------AAHRAGL 375 FDLLDNTDIHLHFPAGAVTKDG PSAGVT IVTCLASLF SGRLVRSDVAMTGE ITLRGLVLPVGGIKDKVL-------AAHRAGL 795 FDLLDNTDIHLHF PAGAVTKDG PSAGVT IVTCLASLF SGRLVRSDVAMTGE ITLRGLVLPVGGIKDKVL-------AAHRAGL 658 FDLLDNTDIHLHF PAGAVTKDG PSAGVT IVTCLASLF SGRLVRSDVAMTGE ITLRGLVLPVGG IKDKVL-------AAHRAGL 524 MNVLDGRDIHIHFPAGAVPKDG PSAGVTLVTALVSLF SQKRVRADTAMTGEMTLRGLVLPVGGIKDKIL-------AAHRYGI 504 NDYLVTSHIHLHVPEGATPKDG PSAGCT IVTALLSLAMGRPVRQNLAMTGEVSLTGK ILPVGG IKEKTI-------AAKRAGV 793 NDYLVTSHIHPHVPEGATPKDGPSAGCA IVTALLSLAMGRPVRQNLAMTGEVSLTGKILPVGGIKEKTI-------AAKRAGV 910 NDELVTSHIHLHVPEGATPKDGPSAGCT IVTALLSLALGQPVLQNLAMTGEVSLTGKVLPVGGIKEKTI-------AAKRAGV 896 NLFLEQEHIHLHVPEGATPKDGPSAG ITIITALVSLATGKPVRQDIAMTGEVSLKGKVLPVGGIKEKTI-------AARRSGV 758 NLFLEOEHIHLHVPEGATPKDGPSAGITIITALVSLATGKPVRODIAMTGEVSLKGKVLPVGGIKEKTI-------AARRSGV 932 NKFF DKAHIHIHVPEGATPKDG PSAGVTLVSSLLSLALDRPVVQDMAMTGE ISLTGKVLPVGG IREKVI-------AARRVGA 930 NQFFANSKLHLHVPAGATPKDG PSAGCTMITSLLSLATKKPVRKDLAMTGEVTLTGRILPIGGVKEKTI-------AARRSQI 894 NOFFANSKLHLHVPAGATPKDGPSAGCTMITSLLSLATKKPVRKDLAMTGEVTLTGRILPIGGVKEKTI-------AARRSQI 384 NLFFANSKLHLHVPEGATPKDGPSAGCTMITSFLSLAMKKLVRKDLAMTGEVTLTGR ILPIGGVKEKTI-------AARRSOI 880 NKLEANSKLHLHVPAGATPKDGPSAGCTMITSLLSLALKKPVRKDLAMTGEVTLTGR ILAIGGVKEKTI-------AARRSQV 898 NRFFEKASIHLHCPEGATPKDG PSAGVTMATSELSLALNKS IDPTVAMTGELTLTGKVLRIGGLREKAV-------AAKRSGA 1067 --TLEDQDIHVHLPAGAVNKDGPSAGTG LACALVSLATNIPLRSDAAVTGE ISLTGHVLPIGGVKEKVL-------AAQREGL 724 GMKPSDYDIHINEPGG-IPIDGPSAG IAMAAG IF SAIHKIPIDNTVAMTGE ISLNGLVKPIGGVIPKIK-------AAKQSGA 497 -----MDIHIQFVGTYEGVEGDSASVS IATAVISAIENIPVDQSVAMTGSLSVRGDVLPVGGVTAKVE-------AAIEAGL 577 GLQMADQDVFVNVVGGVKVTET-SADLALLLAMVS SLRDRPLPQDLVVFGEVGLAGE IRPVPSGQER IS------EAAKHGF 424 -----FSASLTFEQSYSEVDGDSASMAELCALISALADVPVNQSIAITGSVDQFGRAQPVGGLNEKIEGFFAICQQRELTGK 497

(a)

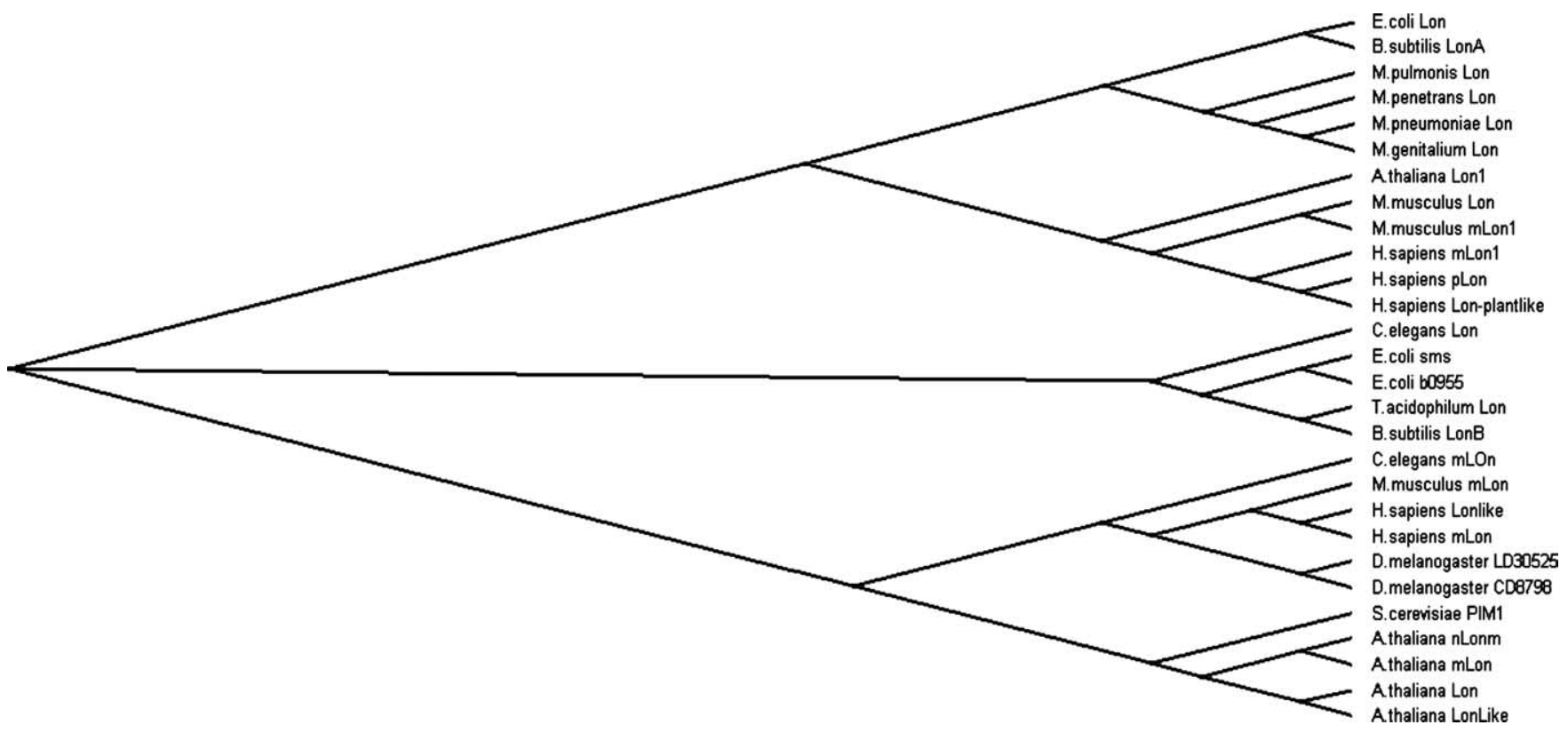

(b)

Fig. 2. Comparison of E. coli Lon orthologs from representative organisms. (a) Multiple sequence alignment of conserved residues surrounding the active site residues of Lon and its orthologs was performed using ClustalW. Serine and lysine residues that form the catalytic dyad are shown in bold. (b) The dendrogram depicts the relatedness between different Lon orthologs. The data from ClustalW was utilized to create the tree using the program TreeView 1.6.6. (http://taxonomy.zoology.gla.ac.uk/rod/rod.html). Accession numbers of orthologs are as follows: A. thaliana_Lon, NP_566258; A. thaliana_Lon1, AAO00734; A. thaliana_Lonlike, NP_566259; A.thaliana_mLon, AAL09722 (m); A.thaliana_nLonm, AAB48000 (m); B. subtilis_LonA NP 390698; B. subtilis_LonB, NP 390699; C. elegans_Lon, NP 499577 (n); C. elegans_mLon, NP 492796 (m); D. melanogaster_LD30525, AAK93211; D. melanogaster_CG8798, AAF49134; E. coli_Lon, AAC73542; E. coli_sms, NP_418806; E. coli_b0955, NP_415475; H. sapiens_mLon, AAA61616 (m); H. sapiens_mLon1, BAB55278; H. sapiens_Lon-plantlike, CAD38889; H. sapiens_Lonlike, CAA52291 (m); H. sapiens_pLon, CAD68987 (p); M. musculus_Lon, BAC34137 (m); M. musculus_mLon, AAN85210 (m); M. musculus_mLon1, BAC35908; M. genitalium_Lon, NP_072905; M. penetrans_Lon, NP_758079; M. pneumoniae_Lon, NP_110020; M. pulmonis_Lon, NP_326350; S. cerevisiae_PIM1, CAA52634; T. acidophilum_Lon, NP_394540. Alphabets in parenthesis after the accession numbers of certain eukaryotic orthologs represent the respective annotated localizations: $\mathrm{m}$, mitochondrial; $\mathrm{n}$, nuclear and p, peroxisomal. 
Table 2

Catalogue of known substrates hydrolyzed by Lon, ClpP and ClpQ

\begin{tabular}{|c|c|c|c|}
\hline Enzyme & EC number & In vitro substrates & Cellular substrates \\
\hline Lon & 3.4 .21 .53 & $\begin{array}{l}\text { Bacteriophage } \lambda \text { protein } \mathrm{N} \\
\text { Casein } \\
\text { CNBr fragments of BSA } \\
\text { Denatured IgG } \\
\text { Glucagon } \\
\text { Oxidized insulin B chain } \\
\text { Fluorogenic peptides, e.g., Suc-AAF-AMC }\end{array}$ & $\begin{array}{l}\text { SulA, RcsA } \\
\text { Bacteriophage } \lambda \text { protein } \mathrm{N} \\
\text { Abnormal/misfolded proteins } \\
\text { Aggregated proteins, aged proteins } \\
\text { Oxidized mitochondrial aconitase } \\
\text { Mutant alkaline phosphatase PhoA61 } \\
\text { UmuC and UmuD, subunits of error prone DNA polymerase } \\
\text { CcrM, an essential DNA methyl transferase in C. crescentus }\end{array}$ \\
\hline ClpP & 3.4 .21 .92 & $\begin{array}{l}\text { Bacteriophage } \lambda \text { protein } \mathrm{O} \\
\beta \text {-Galactosidase fusion proteins } \\
\text { Casein } \\
\text { BSA } \\
\text { Glucagon } \\
\text { SsrA-tagged proteins } \\
\text { Oxidized insulin B chain } \\
\text { Fluorogenic peptides, e.g., Suc-LY-AMC }\end{array}$ & $\begin{array}{l}\text { Proteins induced on C-starvation } \\
\text { Abnormal proteins, canavanylated proteins } \\
\text { Heat shock damaged-proteins } \\
\sigma^{\mathrm{s}} \text {, SsrA-tagged proteins, } \lambda \text { protein } \mathrm{O} \\
\text { ClpA, RepA, Phage P1 PhD } \\
\text { N-end rule substrates } \\
\text { UmuD/UmuD', dimer of error prone DNA polymerase } \\
\text { MazE, endogenous regulator of cell death in E. coli } \\
\text { Dps, DNA protein induced during starvation } \\
\text { DksA, DnaK suppressor protein } \\
\text { C. crescentus CtrA, a key transcriptional regulator } \\
\text { B. subtilis ComK, positive regulator of competence, } \\
\text { CtsR, heat shock regulon repressor, and MurAA, } \\
\text { involved in synthesis of peptidoglycan } \\
\text { Salmonella FlhD and FlhC, regulators of flagellum biosynthesis } \\
\text { Phage Mu transposase and mutated vir repressor }\end{array}$ \\
\hline ClpQ & 3.4.25.- & $\begin{array}{l}\text { Casein } \\
\text { Insulin B chain } \\
\text { Fluorogenic peptides, e.g., Cbz-GGL- } \beta N A\end{array}$ & $\begin{array}{l}\text { SulA } \\
\text { RcsA } \\
\sigma^{32} \\
\text { Abnormal/misfolded proteins } \\
\text { Heat shock damaged-proteins }\end{array}$ \\
\hline
\end{tabular}

archaeabacterium Thermococcus kodakaraensis has been characterized: it utilizes ATP to hydrolyze folded proteins and is membrane-bound [9]. Lactococcus and the eubacterial pathogenic representatives Mycobacterium tuberculosis and $M$. leprae lack lon orthologs in their genomes; however, M. smegmatis, a non-pathogenic relative, harbors Lon [23]. All four Mycoplasma species analyzed harbor Lon orthologs and further biochemical characterization will be required to address whether these Lon orthologs possess proteolytic activity. Interestingly, two Lon paralogs, sms (b4389) and b0955, were detected in the E. coli genome (Fig. 2a) by BLASTP analysis and both possess the conserved catalytic serine of Lon (Fig. 2a); b0955 lines up well with the catalytic lysine whereas sms contains arginine instead of lysine. Both paralogs lack the ATP binding motif (data not shown) and appear to be more similar to Lon from T. acidophilum compared to E. coli (Fig. 2b). Further studies are required to investigate whether they bind to ATPase regulators and function as proteases.

E. coli cells lacking Lon are viable but display well characterized defects, for example, sensitivity to ultraviolet light, methylmethane sulfonate and overproduction of capsular polysaccharide. The absence of Lon leads to the stabilization of SulA and RcsA, which are responsible for the sensitivity to UV and capsular polysaccharide overproduction, respectively. Lon is the most efficient protease in degrading mis- folded proteins in cells lacking DnaK, the heat shock protein (Hsp)-70 ortholog in E. coli [43]. In agreement with these findings, E. coli lacking Lon contains three times more aggregated proteins compared to the wild type [35]. However, overexpression of Lon in E. coli leads to reduced growth due to activation of the YoeB toxin which induces cleavage of translated mRNAs leading to cell death [6].

Lon orthologs appear to have acquired specialized functions in different organisms. A dramatic phenotype is observed in Myxococcus xanthus, a Gram-negative soil bacterium that forms fruiting bodies on nutrient starvation and possesses two Lon paralogs. Lon $\mathrm{V}$ is essential for vegetative growth [41] whereas Lon D is required for fruiting body formation [42]. In the Caulobacter lon mutant, cell division does not go to completion and cell cycle control of initiation of replication is disturbed due to the stabilization of a Lon substrate, an adenine DNA methyltransferase, CcrM [47]. In eukaryotes, Lon plays a major role in mitochondrial function. An increase in expression of Lon leads to a concomitant increase in mitochondrial biogenesis in hepatoma cells [28]. Together, it appears that Lon is an important protease, conserved in different kingdoms. Further elucidation of the crystal structure of full length Lon will be useful in understanding the mechanisms utilized by a protease that harbors both the regulatory and catalytic sites within a single polypeptide. 


\section{Clp}

Clps are barrel-shaped ATP-dependent proteases composed of two components: one catalytic and the other regulatory (Fig. 1b). Two distinct Clp proteolytic subunits of $\sim 23 \mathrm{kDa}$ are known: ClpP and ClpQ (HslV). ClpP is a serine protease, whereas ClpQ belongs to the $\mathrm{N}$-terminal hydrolase family of enzymes with Thr-1 as the active site residue, similar to the $\beta$ subunit of $20 \mathrm{~S}$ proteasomes. Interestingly, catalytically inactive ClpP paralogs, known as ClpR, are found associated with active Clp complexes in cyanobacteria and plants [33,34]. Clp regulators belong to the Clp/Hsp100 family of ATPases, ClpA, B, X and $\mathrm{Y}$ (HslU), and are structurally and functionally distinct. ClpA and ClpB possess two nucleotide binding domains (NBD) whereas ClpX contains a single NBD. ClpY contains an extra domain (I domain) which is inserted into the NBD domain (reviewed in [7]). ClpB exclusively acts as a chaperone whereas $\mathrm{ClpA}$ and $\mathrm{ClpX}$ function as chaperones and also bind to ClpP to form the ATP-dependent proteases ClpAP or ClpXP. ClpY, however, specifically interacts with ClpQ to form the ClpYQ protease. The presence of a tripeptide motif matching (LIVMP)-G-(FL), identified by sequence alignments, mutation analysis and biochemical studies, is an indicator of Clp ATPase binding to ClpP [22]. This motif is present in ClpA and ClpX but is absent in ClpB and ClpY (data not shown), which explains the lack of binding of these regulators to ClpP. The presence of this motif is useful for predicting ClpP binding subunits in other organisms [33]. Three Clp regulators are known in $B$. subtilis: the two NBD containing regulators, $\mathrm{ClpC}$ and ClpE, and the single NBD containing regulator, ClpX. Four distinct ClpP binding regulators are encoded in the Arabidopsis thaliana genome: ClpC, ClpD, ClpS and ClpX [33].

The structure and biochemistry of Clp proteases has been the focus of several studies. ClpP comprises two heptameric rings consisting of an internal central chamber $\sim 50 \AA$ in diameter with 14 serine active sites (Ser-111), one from each subunit, and contains narrow axial pores of $\sim 10 \AA$ diameter for substrate entry [45]. ClpP by itself is able to hydrolyze small peptides but not large peptides or proteins, as access to the proteolytic chamber requires passage through small axial pores. ATP and its nonhydrolyzable forms promote Clp oligomerization (regulator plus protease complexes); however only ATP can support protein unfolding and degradation [36]. ClpA or ClpX forms a single hexameric ring that associates at one or either end of ClpP (Fig. 1b). In fact, ClpP has the potential to form mixed complexes with $\mathrm{ClpA}$ and $\mathrm{ClpX}$ binding to either end to form ClpAPX complexes, which have the potential to bind to a greater range of cellular substrates [14]. ClpA and ClpX bind, unfold and translocate green fluorescent protein-tmRNA encoded peptide tag to ClpP [36]. In fact, unfolding of substrate protein by ClpXP is rate-limiting [21] and requires a lot of energy [20]. ATP-dependent enzymes involved in cytosolic degradation unfold structural elements next to the degradation signal; thus the local structure next to the degradation signal greatly influences the amount of ATP utilized during unfolding of the substrate [20,27]. Cryoelectron microscopy and ClpP mutants were used to demonstrate that substrate proteins bound to distal surfaces followed by accumulation at the inner sides of ATPases and translocation into the catalytic chamber of ClpP [15].

ClpP orthologs are present in some organisms selected for analysis (Table 1). The catalytic triad in E. coli ClpP consists of Ser-111, His-136 and Asp-185 and these residues are conserved in ClpP orthologs (Fig. 3). Most archaeal genomes appear to lack ClpP subunits, including T. acidophilum and S. solfataricus. In addition, M. jannaschii, P. furiosus, Halobacterium sp. NRC-1 and M. mazei lack ClpP orthologs (data not shown). None of the genomes of Mycoplasma species analyzed harbor ClpP orthologs; these are also absent in $S$. cerevisiase and $S$. pombe (data not shown). L. lactis, M. tuberculosis and M. leprae, which lack lon, harbor $c l p P$ orthologs (Table 1; Fig. 3). Interestingly, the genome of $A$. thaliana encodes six catalytically active paralogs of ClpP [33], some of which are included in our analysis (Fig. 3). In A. thaliana, plastid Clp containing complexes are composed of several subunits $(\mathrm{ClpP} 1,3-6)$ whereas the mitochondrial Clp complex is composed of only the ClpP2 subunit [33]. The differential subunit composition of ClpP complexes in plastids and mitochondria in A. thaliana may modulate proteolysis in different organelles.

ClpAP and ClpXP proteases are responsible for several cellular activities and display distinct substrate specificities (reviewed in $[7,17]$ ). In general, ClpAP is able to trap different substrate proteins whereas ClpXP is better at trapping substrate proteins possessing a targeting motif. Using a catalytically inactive ClpP mutant followed by identification of the trapped substrates by mass spectrometry, several ClpP substrates, including DnaK suppressor protein (DksA) and the DNA protective protein induced during starvation (Dps), have been identified. Interestingly, RpoS is the most abundant ClpXP trapped substrate. This strategy led to the identification of at least five distinct signals, present at either the $\mathrm{N}$-terminus or the C-terminus of ClpXP substrates [8]. ClpP complexes are also responsible for degradation of proteins targeted by tmRNA. Functional insights into the tmRNA tagging system have revealed that $\mathrm{ClpA}$ and $\mathrm{ClpX}$ bind to different stretches of the tmRNA encoded peptide tag. Most likely, ClpXP is the major protease involved in degradation of tmRNA encoded peptide tagged proteins in vivo [13].

$\mathrm{ClpP}$ is involved in survival of E. coli during the stationary phase and levels of several proteins (e.g., Dps, 30S ribosomal protein S6 RS-6B etc.) are modulated in E. coli lacking ClpP in the stationary phase [46]. In different organisms $\mathrm{ClpP}$ proteins are known to participate in various physiological processes; for example, it is involved in cell cycle progression from $\mathrm{G} 1$ to $\mathrm{S}$ in Caulobacter [16]. B. subtilis lacking ClpP display multiple defects, including the inability to grow at high temperatures, competence development, 


$$
\begin{aligned}
& \text { E.coli_ClpP } \\
& \text { B.subtilis_ClpP } \\
& \text { H.sapiens_ClpP } \\
& \text { M.musculus_ClpP } \\
& \text { C.elegans_ClpP } \\
& \text { A.thaliana_ClpP1 } \\
& \text { D.melanogaster_CG5045 } \\
& \text { A.thaliana_nClpP1 } \\
& \text { L.lactis_ClpP } \\
& \text { M.tuberculosis_ClpP } \\
& \text { M.leprae_ClpP } \\
& \text { A.thaliana_nClpP3 } \\
& \text { A.thaliana_nClpP4 } \\
& \text { M.tuberculosis_ClpP2 } \\
& \text { M.leprae_ClpP2 }
\end{aligned}
$$

\section{E.coli ClpP}

B.subtilis_ClpP

H.sapiens_ $\bar{C} l p P$

M.musculus ClpP

C.elegans_c̄lpP

A.thaliana ClpP1

D.melanogaster CG5045

A.thaliana_ncl $\bar{p} \mathrm{P} 1$

L.lactis ClpP

M.tubercūosis_ClpP

M. leprae ClpP

A.thaliaña_nClpP3

A.thaliana-nclpP4

M. tuberculosis ClpP2

M.leprae_ClpP2

VSTICMGQAASMGAFLLTAGAKGKRFCLPNSRVMIHQP 138 VSTICIGMAASMGAFLLAAGEKGKRYALPNSEVMIHQP 125 ICTWCVGQAASMGSLLLAAGTPGMRHSLPNSRIMIHQP 180 ICTWCVGQAASMGSLLLAAGS PGMRHSL PNSRIMIHQP 176 VSTWVIGQASSMGSLLLCAGE KGMRSALPNSRIMVHQP 132 ISTICLGQAASMASLLLAAGAKGQRRSLPNATVMIHQP 154 IATWCVGQACSMGSLLLAAGAPGMRYSLPNARIMIHQP 151 VSTVCVGLAASMGAF LLSAGTKGKRYSLPNSRIMIHQP 220 VQTIVMGMAASMGTI IASSGTKGKRFMLPNAEYLIHQP 126 IATYAMGMAASMGEF LLAAGTKGKRYALPHARILMHQP 125 IATYAMGMAA.SMGEF LLAAGTRGKR YALPHARILMHQP 149 VSTVCLGLAASMGAFLLA.SGSKGKRYCMPNSKVMIHQP 191 VSTIALGIAASTAS I ILGAGTKGKRF AMPNTRIMIHQP 185 IQTVCLGQAASAAAVLLAAGTPGKRMALPNARVLIHQP 137 IQTVCLGQAASAAAVLLAAGTPGKRMALPNARVLIHQP 137

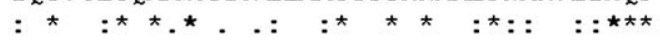

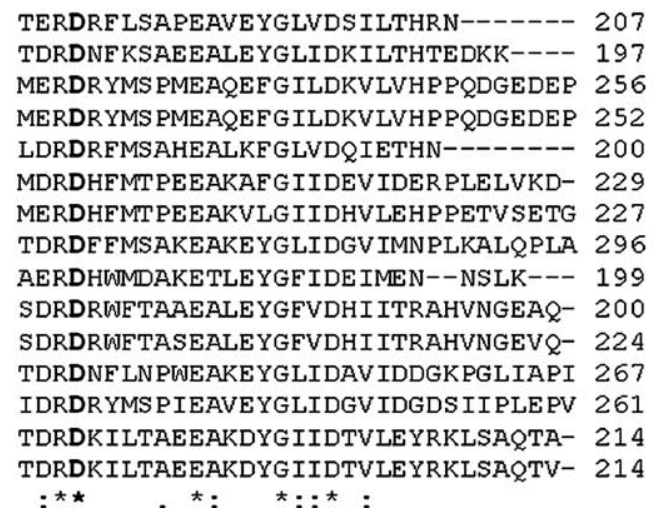

Fig. 3. Alignment of sequences flanking the active site residues in ClpP orthologs. Multiple sequence alignment of mouse ClpP orthologs surrounding the three catalytic residues (bold) from representative organisms was performed using ClustalW. The residues important for activity are shown in bold. Accession numbers of the corresponding orthologs are as follows: A. thaliana_ClpP1, BAC43126; A. thaliana_nClpP1, BAA82065; A. thaliana_nClpP3, AAL66941; A. thaliana_nClpP4, AAK68772; B. subtilis_ClpP, AAC46381; C. elegans_ClpP, NP_496215; D. melanogaster_CG5045, NP_609388; E. coli_ClpP, AAC73540; H. sapiens_ClpP, AAH02956; L. lactis_ClpP, NP_266829; M. leprae_ClpP, CAC30430; M. leprae_ClpP2, CAC30429; M. musculus_ClpP, NP_059089 (annotated mitochondrial localization); M. tuberculosis_ClpP, CAA16038; M. tuberculosis_ClpP2, CAA16037.

motility and sporulation [31]. ClpP orthologs are essential in C. reinhardtii [29], the cyanobacterium Synechococcus [34] and tobacco [25]. Although both mice and humans possess a ClpP ortholog (Table 1), the function of this ClpP has not been identified.

ClpYQ (HslUV) was identified as a heat shock operon with similarity to Clp ATPases and 20S proteasomal $\beta$ subunits. ClpY and ClpQ can exist separately and ATP is required for oligomerization of the ClpYQ complex and protease activity. The crystal structures of ClpYQ [37] revealed a close resemblance with $20 \mathrm{~S}$ proteasomes with a similar subunit structure and intersubunit interactions. However, 20S proteasomes are composed of heptameric rings, whereas ClpYQ are composed of hexameric rings (Fig. 1b). The unique I-domain in ClpY is thought to extend outward from the complex and assist in the binding of substrates; however, ClpY lacking the I-domain can form intact complexes that are indistinguishable from wild type ClpYQ and display proteolytic activity [26]. Further studies are required to investigate the functional roles of I domainlacking ClpYQ complexes in vivo. The N-terminal catalytic Thr- 1 of ClpQ, similar to the $20 \mathrm{~S}$ proteasomal $\beta$ subunit, is the active site nucleophile [50]. Quite surprisingly, ClpYQ of B. subtilis (CodWX) utilizes a serine residue present prior to the double threonines as the active site residue. In fact, this enzyme is the only known N-terminal serine protease and mutation of this serine residue to alanine or even threonine abolishes the peptidase activity, although the ATPase activity remains intact. Interestingly, mutation of double threonines resulted in inability to form the tetradecamer, resulting in loss of peptidase activity as well as ATPase activity [18]. Biochemical studies and electron micrographs have revealed that ClpYQ from B. subtilis is distinct from its E. coli counterpart [19].

Of the representative organisms selected for the present analysis, only $E$. coli and $B$. subtilis encoded $\operatorname{clp} Q$ sequences (Table 1). It was thought that 20S proteasomes and ClpYQ are mutually exclusive; however, genomes of several microbes are now known to harbor both $20 \mathrm{~S}$ proteasomes and ClpYQ or lack both these proteases [11]. Significantly, ClpYQ overexpression can rescue lon-deficient cells by hydrolyzing most Lon substrates, including SulA, RcsA and $\mathrm{RpoH}$ [48]. Together, Clp proteases possess specialized functions in various organisms. However, most studies on Clp complexes have been performed on lower organisms. Further studies are required to identify cellular substrates and functional roles of $\mathrm{ClpP}$ in organelles of plants and animals. 


\section{Conclusions}

The current genomic survey and review of the literature has revealed certain interesting features: (i) It had been argued previously that common genes present in two small bacterial genomes, Mycoplasma and H. influenzae, represent genes important in cellular metabolism [32]. Lon appears to be the main ATP-dependent enzyme in Mycoplasma. However, $H$. influenzae lacks Lon but contains both ClpXP and ClpYQ (data not shown). In our analysis, S. solfataricus is the only organism lacking both ClpP and Lon orthologs. An AAA-ATPase (NP 343775) from Sulfolobus displays significant similarity (E value-1e-05) with Lon, although the active site residues could not be aligned. It is possible that this potential Lon ortholog has diverged and further functional characterization of this enzyme is required to ascertain whether it can function as a protease. However, proteasome-activating nucleotidase (PAN), an AAA-ATPase family member, and $20 \mathrm{~S}$ proteasomes are both present in S. solfataricus (data not shown) and may play an important role during ATP-dependent cytosolic protein degradation in this organism. This clearly indicates that, in principle, at least one ATP-dependent enzyme is encoded in genomes from different kingdoms. (ii) Both chaperones and proteases are known to be induced in E. coli during heat shock. Further genetic and biochemical studies are required to identify cellular protein substrates and decipher the roles of individual protease orthologs during different stress conditions. (iii) E. coli proteases (Clp and Lon), unlike their eukaryotic counterparts (26S proteasomes), are not essential. However, these proteases may be essential under some conditions or in some organisms (discussed above). E. coli lacking ClpP and Lon are unable to survive the transition from growth in rich media to minimal media, a form of stress known as nutritional downshift [24]. E. coli lacking Lon is impaired in degradation of puromycyl peptides. However, this effect was not observed in M. smegmatis lacking Lon and $20 \mathrm{~S}$ proteasomes [23]. The relevance of two ClpP paralogs in pathogens, e.g., M. tuberculosis and M. leprae, both lacking Lon (Table 1; Fig. 3), remains to be evaluated. Further functional studies are required to understand the roles of protease orthologs in different organisms. (iv) One of the interesting aspects that have emerged recently is the role of enzymes involved in cytosolic protein degradation in pathogenesis. $\operatorname{clp} P$ and $\operatorname{clpX}$-deficient $S$. typhimurium strains are unable to form colonies in vivo due to impaired ability to grow intracellularly in macrophages [49]. Also, ClpP is required for virulence in Listeria monocytogenes [10]. S. typhimurium lacking lon express high levels of proteins encoded by Salmonella pathogenicity island (SPI)-I genes, demonstrating that Lon is a negative regulator $[3,39]$. The failure of lon deficient $S$. typhimurium to downmodulate SPI-I and upregulate SPI-II genes is probably responsible for the inability of these cells to cause systemic infection in a mouse model of infection [40]. These results have im- plicated Lon- or ClpP-lacking strains as candidate vaccine targets $[10,30]$.

In summary, the complete genome sequences of different organisms offer an opportunity to experimentally explore the roles of newer enzymes and their orthologs in the field of cytosolic protein degradation. As ATP-dependent proteases play key roles in initiating the process of cytosolic protein degradation, further experimental verification using a systematic deletion strategy needs to evaluate the roles of protease orthologs in growth (in vitro and in vivo), different stress conditions and pathogenesis. Studies on these enzymes will reveal their distinct functions in different organelles and organisms and will consequently enrich this area of research.

\section{Acknowledgements}

We thank Anujith Kumar and Pankaj Tahiliani for their help with preparation of this manuscript. The comments of researchers in the field of E. coli cytosolic protein degradation are greatly appreciated. Only selected references were incorporated due to limitations of space and we apologize to researchers whose contributions were not cited. This study has been supported by a grant from the Department of Science and Technology, Government of India.

\section{References}

[1] D. Biran, E. Gur, L. Gollan, E.Z. Ron, Control of methionine biosynthesis in Escherichia coli by proteolysis, Mol. Microbiol. 37 (2000) 1436-1443.

[2] C. Birghan, E. Mundt, A.E. Gorbalenya, A non-canonical lon proteinase lacking the ATPase domain employs the Ser-Lys catalytic dyad to exercise broad control over the life cycle of a double-stranded RNA virus, EMBO J. 19 (2000) 114-123.

[3] J.D. Boddicker, B.D. Jones, Lon protease activity causes downregulation of Salmonella pathogenicity island 1 invasion gene expression after infection of epithelial cells, Infect. Immun. 72 (2004) 20022013.

[4] I. Botos, E.E. Melnikov, S. Cherry, J.E. Tropea, A.G. Khalatova, F. Rasulova, Z. Dauter, M.R. Maurizi, T.V. Rotanova, A. Wlodawer, A. Gustchina, The catalytic domain of E. coli Lon protease has a unique fold and a Ser-Lys dyad in the active site, J. Biol. Chem. 279 (2004) 8140-8148.

[5] D. Chandu, D. Nandi, From proteins to peptides to amino acids: Comparative genomics of enzymes involved in downstream processing events during cytosolic protein degradation, Appl. Gen. Proteom. 1 (2002) 235-252.

[6] S.K. Christensen, G. Maenhaut-Michel, N. Mine, S. Gottesman, K. Gerdes, L. Van Melderen, Overproduction of the Lon protease triggers inhibition of translation in Escherichia coli: Involvement of the yefM-yoeB toxin-antitoxin system, Mol. Microbiol. 51 (2004) 1705-1717.

[7] D.A. Dougan, A. Mogk, B. Bukau, Protein folding and degradation in bacteria: To degrade or not to degrade? That is the question, Cell Mol. Life Sci. 59 (2002) 1607-1616.

[8] J.M. Flynn, S.B. Neher, Y.I. Kim, R.T. Sauer, T.A. Baker, Proteomic discovery of cellular substrates of the ClpXP protease reveals five classes of ClpX-recognition signals, Mol. Cell 11 (2003) 671-683. 
[9] T. Fukui, T. Eguchi, H. Atomi, T. Imanaka, A membrane-bound archaeal Lon protease displays ATP-independent proteolytic activity towards unfolded proteins and ATP-dependent activity for folded proteins, J. Bacteriol. 184 (2002) 3689-3698.

[10] O. Gaillot, E. Pellegrini, S. Bregenholt, S. Nair, P. Berche, The $\mathrm{ClpP}$ serine protease is essential for the intracellular parasitism and virulence of Listeria monocytogenes, Mol. Microbiol. 35 (2000) 12861294.

[11] C. Gille, A. Goede, C. Schloetelburg, R. Preissner, P.M. Kloetzel, U.B. Gobel, C. Frommel, A comprehensive view on proteasomal sequences: Implications for the evolution of the proteasome, J. Mol. Biol. 326 (2003) 1437-1448.

[12] R. Gillet, B. Felden, Emerging views on tmRNA-mediated protein tagging and ribosome rescue, Mol. Microbiol. 42 (2001) 879-885.

[13] S. Gottesman, E. Roche, Y.N. Zhou, R.T. Sauer, The ClpXP and ClpAP proteases degrade proteins with carboxy-terminal peptide tails added by the SsrA-tagging system, Gen. Dev. 12 (1998) 1338-1347.

[14] J. Ortega, H.S. Lee, M.R. Maurizi, A.C. Steven, ClpA and ClpX ATPases bind simultaneously to opposite ends of ClpP peptidase to form active hybrid complexes, J. Struct. Biol. 146 (2004) 217-226.

[15] T. Ishikawa, F. Beuron, M. Kessel, S. Wickner, M.R. Maurizi, A.C. Steven, Translocation pathway of protein substrates in ClpAP protease, Proc. Natl. Acad. Sci. USA 98 (2001) 4328-4333.

[16] U. Jenal, T. Fuchs, An essential protease involved in bacterial cellcycle control, EMBO J. 17 (1998) 5658-5669.

[17] U. Jenal, R. Hengge-Aronis, Regulation of proteolysis in bacterial cells, Curr. Opin. Microbiol. 6 (2003) 163-172.

[18] M.S. Kang, B.K. Lim, I.S. Seong, J.H. Seol, N. Tanahashi, K. Tanaka, C.H. Chung, The ATP-dependent CodWX (HslVU) protease in Bacillus subtilis is an N-terminal serine protease, EMBO J. 20 (2001) 734742.

[19] M.S. Kang, S.R. Kim, P. Kwack, B.K. Lim, S.W. Ahn, Y.M. Rho, I.S. Seong, S.C. Park, S.H. Eom, G.W. Cheong, C.H. Chung, Molecular architecture of the ATP dependent CodWX protease having an N-terminal serine active site, EMBO J. 22 (2003) 2893-2902.

[20] J.A. Kenniston, T.A. Baker, J.M. Frenandez, R.T. Sauer, Linkage between ATP consumption and mechanical unfolding during the protein processing reactions of an $\mathrm{AAA}+$ degradation machine, Cell 114 (2003) 511-520.

[21] Y.I. Kim, R.E. Burton, B.M. Burton, R.T. Sauer, T.A. Baker, Dynamics of substrate denaturation and translocation by the ClpXP degradation machine, Mol. Cell 5 (2000) 639-648.

[22] Y.I. Kim, I. Levchenko, K. Fraczkowska, R.V. Woodruff, R.T. Sauer, T.A. Baker, Molecular determinants of complex formation between Clp/Hsp100 ATPases and the ClpP peptidase, Nat. Struct. Biol. 8 (2001) 230-233.

[23] N. Knipfer, A. Seth, S.G. Roudiak, T.E. Shrader, Species variation in ATP-dependent protein degradation: Protease profiles differ between mycobacteria and protease functions differ between Mycobacterium smegmatis and Escherichia coli, Gene 231 (1999) 95-104.

[24] A. Kuroda, K. Nomura, R. Ohtomo, J. Kata, T. Ikeda, N. Takiguchi, H. Ohtake, A. Kornberg, Role of inorganic phosphate in promoting ribosomal protein degradation by the lon protease in E. coli, Science 293 (2001) 705-708.

[25] H. Kuroda, P. Maliga, The plastid clpP1 protease gene is essential for plant development, Nature 425 (2003) 86-89.

[26] A.R. Kwon, B.M. Kessler, H.S. Overkleeft, D.B. McKay, Structure and reactivity of an asymmetric complex between HslV and I-domain deleted HslU, a prokaryotic homologue of the eukaryotic proteasome, J. Mol. Biol. 330 (2003) 185-195.

[27] C. Lee, M.P. Schwartz, S. Prakash, M. Iwakura, A. Matouschek, ATPdependent proteases degrade their substrates by processively unraveling them from the degradation signal, Mol. Cell 7 (2001) 627-637.

[28] K. Luciakova, B. Sokolikova, M. Chloupkova, B.D. Nelson, Enhanced mitochondrial biogenesis is associated with increased expression of the mitochondrial ATP-dependent Lon protease, FEBS Lett. 444 (1999) 186-188.
[29] C. Huang, S. Wang, L. Chen, C. Lemieux, C. Otis, M. Turmel, X.Q. Liu, The Chlamydomonas chloroplast clpP gene contains translated large insertion sequences and is essential for cell growth, Mol. Gen. Genet. 244 (1994) 151-159.

[30] H. Matsui, M. Suzuki, Y. Isshiki, C. Kodama, M. Eguchi, Y. Kikuchi, K. Motokawa, A. Takaya, T. Tomoyasu, T. Yamamoto, Oral immunization with ATP-dependent protease-deficient mutants protects mice against subsequent oral challenge with virulent Salmonella enterica serovar typhimurium, Infect. Immun. 71 (2003) 30-39.

[31] T. Msadek, V. Dartois, F. Kunst, M.L. Herbaud, F. Denizot, G. Rapoport, ClpP of Bacillus subtilis is required for competence development, motility, degradative enzyme synthesis, growth at high temperature and sporulation, Mol. Microbiol. 27 (1998) 899-914.

[32] A.R. Mushegian, E.V. Koonin, A minimal gene set for cellular life derived by comparison of complete bacterial genomes, Proc. Natl. Acad. Sci. USA 93 (1996) 10268-10273.

[33] J.-B. Peltier, D.R. Ripoll, G. Friso, A. Rudella, Y. Cai, J. Ytterber, L. Giacomelli, J. Pillardy, K.J. van Wijk, Clp protease complexes from photosynthetic and non-photosynthetic plastids and mitochondria of plants, their predicted three-dimensional structures, and functional implications, J. Biol. Chem. 279 (2004) 4768-4781.

[34] J. Schelin, F. Lindmark, A.K. Clarke, The $\operatorname{clpP}$ multigene family for the ATP-dependent Clp protease in the cyanobacterium Synechoccous, Microbiology 148 (2002) 2255-2265.

[35] R. Rosen, D. Biran, E. Gur, D. Becher, M. Hecker, E.Z. Ron, Protein aggregation in Escherichia coli: Role of proteases, FEMS Microbiol. Lett. 207 (2002) 9-12.

[36] S.K. Singh, R. Grimaud, J.R. Hoskins, S. Wickner, M.R. Maurizi, Unfolding and internalization of proteins by the ATP-dependent proteases ClpXP and ClpAP, Proc. Natl. Acad. Sci. USA 97 (2000) 8898-8903.

[37] M.C. Sousa, C.B. Trame, H. Tsuruta, S.M. Wilbanks, V.S. Reddy, D.B. McKay, Crystal and solution structures of an HslUV proteasechaperone complex, Cell 103 (2000) 633-643.

[38] H. Stahlberg, E. Kutejova, K. Suda, B. Wolpensinger, A. Lustig, G. Schatz, A. Engel, C.K. Suzuki, Mitochondrial Lon of Saccharomyces cerevisiae is a ring-shaped protease with seven flexible subunits, Proc. Natl. Acad. Sci. USA 96 (1999) 6787-6790.

[39] A. Takaya, T. Tomoyasu, A. Tokumitsu, M. Morioka, T. Yamamoto, The ATP-dependent lon protease of Salmonella enterica serovar Typhimurium regulates invasion and expression of genes carried on Salmonella pathogenicity island 1, J. Bacteriol. 184 (2002) 224-232.

[40] A. Takaya, M. Suzuki, H. Matsui, T. Tomoyasu, H. Sashinami, A. Nakane, T. Yamamoto, Lon, a stress-induced ATP-dependent protease, is critically important for systemic Salmonella enterica serovar typhimurium infection of mice, Infect. Immun. 71 (2003) 690-696.

[41] N. Tojo, S. Inouye, T. Komano, Cloning and nucleotide sequence of the Myxococcus xanthus lon gene: Indispensability of lon for vegetative growth, J. Bacteriol. 175 (1993) 2271-2277.

[42] N. Tojo, S. Inouye, T. Komano, The lon $D$ gene is homologous to the lon gene encoding an ATP-dependent protease and is essential for the development of Myxococcus xanthus, J. Bacteriol. 175 (1993) 4545-4549.

[43] T. Tomoyasu, A. Mogk, H. Langen, P. Goloubinoff, B. Bukau, Genetic dissection of the roles of chaperones and proteases in protein folding and degradation in the Escherichia coli cytosol, Mol. Microbiol. 40 (2001) 397-413.

[44] L. Van Melderen, S. Gottesman, Substrate sequestration by a proteolytically inactive Lon mutant, Proc. Natl. Acad. Sci. USA 96 (1999) 6064-6071.

[45] J. Wang, J.A. Hartling, J.M. Flanagan, The structure of ClpP at $2.3 \AA$ resolution suggests a model for ATP-dependent proteolysis, Cell 91 (1997) 447-456.

[46] D. Weichart, N. Querfurth, M. Dreger, R. Hengge-Aronis, Global role for ClpP-containing proteases in stationary-phase adaptation of Escherichia coli, J. Bacteriol. 185 (2003) 115-125. 
[47] R. Wright, C. Stephens, G. Zweiger, L. Shapiro, M.R. Alley, Caulobacter Lon protease has a critical role in cell-cycle control of DNA methylation, Gen. Dev. 10 (1996) 1532-1542.

[48] W.F. Wu, Y. Zhou, S. Gottesman, Redundant in vivo proteolytic activities of Escherichia coli Lon and the ClpYQ (HslUV) protease, J. Bacteriol. 181 (1999) 3681-3687.

[49] T. Yamamoto, H. Sashinami, A. Takaya, T. Tomoyasu, H. Matsui, Y. Kikuchi, T. Hanawa, S. Kamiya, A. Nakane, Disruption of the genes for ClpXP protease in Salmonella enterica serovar Typhimurium results in persistent infection in mice, and development of persistence requires endogenous gamma interferon and tumor necrosis factor alpha, Infect. Immun. 69 (2001) 3164-3174.

[50] S.J. Yoo, Y.K. Shim, I.S. Seong, J.H. Seol, M.S. Kang, C.H. Chung, Mutagenesis of two N-terminal Thr and five Ser residues in HslV, the proteolytic component of the ATP-dependent HsIVU protease, FEBS Lett. 412 (1997) 57-60. 